\title{
Impact of migration origin on individual protection strategies against sexual transmission of HIV in Paris metropolitan area, SIRS cohort study, France
}

Thomas Kesteman ${ }^{1,2^{*}}$, Annabelle Lapostolle ${ }^{1}$, Dominique Costagliola ${ }^{1}$, Véronique Massari ${ }^{1}$ and Pierre Chauvin ${ }^{1}$

\begin{abstract}
Background: The impact of migration and country or region of origin on sexual behaviours and prevention of the sexual transmission of HIV has been scarcely studied in France. The objective of this study was to evaluate if and how individual attitudes of prevention towards HIV infection are different according to country or region of origins in Paris area, France.

Methods: 3006 individuals were interviewed in the Paris metropolitan area in 2010. Outcome variables were (i) the intention of the individual to protect oneself against HIV, and (ii) the adoption of a condom-based approach for protection against HIV. To explore factors associated with these outcomes, we constructed multivariate logistic regression models, first taking into account only demographic variables -including country of origin-, then successively adding socioeconomic variables and variables related to sexual behaviour and HIV perception and prevention behaviour.

Results: French and foreign people who have origins in Sub-Saharan Africa declared more intentions to protect themselves than French people with French parents (in foreign men, $\mathrm{aOR}=3.43$ [1.66-7.13]; in foreign women, $\mathrm{aOR}=2.94$ [1.65-5.23]), but did not declare more recourse to a condom-based approach for protection against HIV (in foreign men, $\mathrm{aOR}=1.38$ [0.38-4.93]; in foreign women, $\mathrm{aOR}=0.93$ [0.40-2.18]). Conversely, foreign women and French women from foreign origin, especially from Maghreb (Northern Africa), reported less intention of protection than French women with French parents.

Conclusions: These results underline the importance of taking culture and origins of target populations into consideration when designing information, education and communication about HIV and sexually transmitted diseases. These results also draw attention to fractions of the general population that could escape from prevention messages.
\end{abstract}

\section{Background}

In our globalized world, it is now well known that health impacts migration and reversely, migration impacts health $[1,2]$. Similarly, ethnicity or geographical origins influence health and health behaviours [3], and more specifically, migration and origins affect sexual knowledge

\footnotetext{
* Correspondence: kesteman@agora.eu.org

${ }^{1}$ Department of Social Epidemiology, Sorbonne Universités, UPMC Univ Paris 06, INSERM, Institut Pierre Louis d'Epidémiologie et de Santé Publique (IPLESP UMRS 1136), F75013 Paris, France

${ }^{2}$ Institut Pasteur de Madagascar, BP 1274 Avaradoha, Antananarivo 101, Madagascar
}

and behaviours [4-6]. In France, immigrants come principally from Southern and Eastern Europe and from former French colonies, mainly Maghreb (Northern Africa) and Sub-Saharan Africa (SSA) [7, 8], including therefore a significant proportion of people originating from countries with high HIV prevalence [9]. Also, in France as in many other European countries, immigrants are disproportionally represented among the poor and the socially excluded populations [10]; which can make them highly vulnerable to HIV/AIDS $[9,11]$. Among this migrant population, an important part live in Paris and its suburbs (the capital region), where they now account for $20.2 \%$ of the 
population [8]. In France in 2010, $57.2 \%$ of new HIV diagnoses were due to heterosexual transmission and $47.7 \%$ occurred in people born abroad [12]. Although the proportion of people born abroad among new HIV diagnoses decreased during the last decade in France, migrants are still disproportionately affected by the epidemic, in particular people from SSA who accounted for $31.7 \%$ of new HIV diagnoses in France in 2010 [12]. Since 2001, migrants are considered as a priority for control programs by the French health authorities [13, 14], and targeted prevention interventions have been conducted [15-17], but few or none has been reported in the scientific English literature [11].

Prevention practices against the HIV infection are, at least partially, driven by intended or unconscious attitudes that take into account individuals' health knowledge as well as their social values [18-20] and both of them are different according to country of origin [20]. More generally, the social determinants of these practices include people's demographics (sex, age, country of origin), their relationship characteristics (duration, gender values, communication, sexual practice), their social norms (which varied according to their degree of integration into social groups), and the social context of a given intercourse $[4-6,18,19,21,22]$. In France, the relationship between country of origin and HIV protection attitudes and behaviours has been scarcely statistically studied [22], partly because of the existing legal restrictions in collecting and processing data regarding ethnicity or migration status [1, 23-27].

The aim of this study was to evaluate whether or not individual attitudes of prevention are different according to country of origin in the Paris metropolitan area. More specifically, our objectives were to investigate whether relationships exist between people's origin and (i) their intention of protection against HIV, and (ii) their adoption of a condom-based approach for HIV protection.

\section{Methods}

\section{Study sample}

The SIRS cohort (a French acronym for Health, Inequalities and Social Ruptures) is the first large, representative, population-based French cohort set up to study the social determinants of health and health-care utilization in the field of social epidemiology [28-31]. In 2005, at inclusion, our study population was a multistagerandom sample of the adult French-speaking population living in the Paris metropolitan area (Paris and the 3 adjacent departments, a region of 8.7 million inhabitants). The primary sampling units were census blocks of about 2000 inhabitants each: 50 of them were randomly selected from the 2595 eligible census blocks in Paris and its suburbs, according to their socioeconomic situation. Sixty households were then chosen at random in each census block. Lastly, one adult in each household was randomly selected using the next-birthday method and face-to-face interviewed, using a questionnaire that gathers more than 400 variables related to economic status, social integration, health, health-related behaviours, and the use of the health-care system. This cohort study had received ethical and legal authorization from two national authorities for non-biomedical researches: the Comité consultatif sur le traitement de l'information en matière de recherche dans le domaine de la santé (CCTIRS) and the Commission nationale de l'informatique et des libertés (CNIL).

In 2010, $47.2 \%$ of participants included in 2005 were interviewed again [32]; $0.6 \%$ had died, $1.5 \%$ were too sick to participate, $3.6 \%$ had moved outside the selected census blocks, $8.5 \%$ were not present at the time of the survey, $15.7 \%$ refused to participate again and $22.9 \%$ were lost to follow-up without information. People who could not be re-interviewed in 2010 were replaced by other people of the same census block, following a random methodology identical to the one described above. As in 2005, refusal rate in 2010 was $29.1 \%$. Sex ratio and mean age of people followed up were not different from newly included people. This study analyzed the data collected in of 2010 among the final sample of 3006 individuals.

\section{Variables \\ Intention of protection against HIV and declaration of a condom-based approach for HIV protection}

Two outcomes have been successively studied. The intention of protection against HIV corresponds to the positive answer to the question "Do you usually do something to protect yourself against HIV?" If yes, the interviewee was then asked "Personally, what do you do to protect yourself against HIV?"; if no, he/she was asked for what reasons he/she did not do so. These two latter questions could be answered the same ways by choosing one or several of the following items: steady use of condoms, abstinence, fidelity, trust, HIV testing, dialog with partner, restricting sexual intercourse to people of the same community, religious faith, none. This questionnaire design allowed us to distinguish, for a given behaviour or attitude (e.g. using condoms), between intended and unintended protective means against HIV infection. We considered that a person adopted a 'condom-based approach' when he/she mentioned at least the steady use of condoms as a means of protection against HIV (the term "steady use" being self-defined and self-declared, without any further precision).

\section{Migration origin}

In this study, we defined participants' "migration origin" on the basis of both their nationality and their parents' 
nationality [33], and we distinguished French people with French parents (the majority of the population) from French people of SSA origin (i.e. French, born to at least one parent with a SSA nationality), foreigners from SSA, French of Maghrebi origin (Maghreb including Mauritania, Morocco, Algeria, Libya, and Tunisia), foreigners from Maghreb, French of European origin, foreigners from Europe, French from other origins, and foreigners from other countries. When parents had different foreign citizenships (in $3.3 \%$ of the cases), the father's one was the default citizenship considered. All the different categories of French people with foreign origins may include both people born French from foreign parents, and people born foreigner but who subsequently acquired the French citizenship (whatever their country of birth was - in France or abroad - in both cases); making the simplifying assumption that all these situations correspond to a similar level of acculturation [4].

\section{Covariables}

The association between migration origin and each of our two outcomes was estimated, first only adjusted on age and, then, when further taking into account some behavioural and social characteristics that could play the role of effect modifiers or intermediary factors (Table 1): socioeconomic factors (education and income), religious practice, variables related to sexual biography (couple status, lifetime number of couple relationships, number of sexual partners in the last 5 years, self-perceived sexual orientation), variables related to communication about sexuality and perception of HIV (intimate social network, perceived susceptibility to infection by HIV, declared stigma toward HIV+ people), and variables related to HIV prevention behaviours (use of condoms, voluntary HIV testing). All covariables were tested for univariate association with outcome variables.

The intimate social network was defined by the number of persons to whom individuals speak about sexuality. Regarding stigma towards $\mathrm{HIV}+$ people, we distinguished participants expressing no stigma at all (i.e. who would accept working with a HIV+ person, having a meal at his/ her home, going on leave with him/her, leaving him/her looking after his/her children or grand-children, and having sex with him/her using a condom) from those expressing stigma (i.e. who would not accept at least one of the situations listed above). The perceived susceptibility to HIV infection was asked by a single Yes/No question: "Have you ever feared being infected by AIDS virus?" The questionnaire was based on previous studies [28-31, 34, 35] and pre-tested.

\section{Statistical analyses}

Analyses were performed on 2994 persons, excluding HIV positive people $(n=8$, as declared either in an open list of "serious and/or chronic" medical conditions, or in the complete list of the chronic conditions fully covered by the French Social security health insurance, in two other parts of the SIRS questionnaire) and non-response $(n=4)$. First, we examined the prevalence of the intention of protection against HIV and the adoption of a condom-based approach for protection against HIV by country of origin and according to all the covariates (Table 1). All the numbers and proportions presented in Table 1 were weighted to account for the complex sample design and for the post-stratification adjustment on age and gender, according to the 2006 census data in Paris area, as described elsewhere [29].

To explore factors associated with the intention of protection and the adoption of a condom-based approach for protection against HIV, we estimated logistic regression models, taking into account the cluster effect (vce(cluster)) in Stata $11^{\circ}$ software, separately for men and women (as sexual biographies, attitudes and behaviours usually differ between men and women [19, 36]). After estimating the association between migration origin and both outcomes with participants' age as the only covariables (which was preferred to an univariate model since age influences strongly perceptions and behaviours related to sex), we estimated five models, adding successively variables related to 1) demographics, 2) socioeconomic status, 3) sexual biography, 4) communication about sexuality and perception of HIV, and 5) HIV prevention behaviours. Only covariables significantly associated with at least one outcome (in men or in women) were retained at each step. For simplicity of presentation, only the first models (adjusted on age only) and the final ones are presented here (respectively named model 1 and model 2 in Tables 2 and 3) but all intermediate models can be found in Additional file 1 .

Finally, we conducted a mediation analysis in order to disentangle effects of stigma and perceived susceptibility to HIV infection on the intention of protection against HIV.

\section{Results}

\section{Individual characteristics}

Altogether $87.4 \%$ of the study population was French, $3.2 \%$ was Sub-Saharan African and $2.9 \%$ was Maghrebi (Table 1). Among the French citizens, $24.7 \%$ were of foreign origin according to our definition; this included migrants who acquired French citizenship (53.6 \% of French with foreign origins) together with French-born, non-migrants who were born in France from foreign parents.

\section{Intention of protection}

About one third of the sample (31.0\%) stated that they protected themselves from HIV (Table 1). Men were 
Table 1 Prevalence of the intention of protection against HIV and the adoption of a condom-based approach for protection against HIV by subgroups. Paris metropolitan area, France, 2010

\begin{tabular}{|c|c|c|c|c|c|c|c|c|c|}
\hline \multirow[b]{2}{*}{ Variables } & \multirow[b]{2}{*}{ Category } & \multicolumn{4}{|c|}{$\begin{array}{l}\text { Intention of protection } \\
\text { against HIV }\end{array}$} & \multicolumn{4}{|c|}{ Adoption of a condom-based approach } \\
\hline & & Men & $\%$ & Women & $\%$ & Men & $\%$ & Women & $\%$ \\
\hline \multicolumn{10}{|l|}{ Demographic variables } \\
\hline \multirow[t]{9}{*}{ Migration origin } & French with French parents & $372 / 939$ & 39.6 & $315 / 1030$ & 30.6 & $330 / 939$ & 35.1 & $220 / 1030$ & 21.4 \\
\hline & French of Sub-Saharan origin & $34 / 58$ & 58.3 & $21 / 55$ & 38.6 & $24 / 58$ & 41.0 & $14 / 55$ & 24.8 \\
\hline & Sub-Saharan foreigner & $31 / 45$ & 68.1 & $28 / 50$ & 56.2 & $22 / 45$ & 48.9 & $18 / 50$ & 35.7 \\
\hline & French of Maghrebi origin & $34 / 89$ & 37.9 & 23/111 & 20.6 & 23/89 & 25.9 & $14 / 111$ & 12.9 \\
\hline & Maghrebi foreigner & $20 / 46$ & 43.0 & $5 / 41$ & 13.0 & $14 / 46$ & 30.4 & $6 / 41$ & 14.7 \\
\hline & French of European origin & $32 / 73$ & 43.6 & $23 / 110$ & 21.0 & $29 / 73$ & 40.4 & $15 / 110$ & 14.1 \\
\hline & European foreigner & $15 / 61$ & 24.0 & $14 / 60$ & 23.8 & $14 / 61$ & 22.7 & $8 / 60$ & 13.6 \\
\hline & French of other origin & $18 / 57$ & 32.6 & $28 / 95$ & 29.3 & $12 / 57$ & 20.9 & $18 / 95$ & 18.9 \\
\hline & Other foreigner & $13 / 36$ & 37.0 & $6 / 39$ & 15.5 & $7 / 36$ & 18.8 & $7 / 39$ & 17.2 \\
\hline \multirow[t]{4}{*}{ Age } & $\leq 29$ years old & $233 / 300$ & 77.8 & $163 / 298$ & 54.7 & $227 / 300$ & 75.9 & $137 / 298$ & 45.9 \\
\hline & $30-44$ years old & $203 / 451$ & 45.1 & $174 / 492$ & 35.3 & $154 / 451$ & 34.3 & $113 / 492$ & 23.0 \\
\hline & $45-59$ years old & $91 / 334$ & 27.4 & $93 / 381$ & 24.5 & $66 / 334$ & 19.7 & $57 / 381$ & 15.1 \\
\hline & $\geq 60$ years old & $39 / 318$ & 12.4 & $33 / 419$ & 7.9 & $27 / 318$ & 8.3 & $13 / 419$ & 3.0 \\
\hline \multicolumn{10}{|l|}{ Socio-economic variables } \\
\hline \multirow[t]{3}{*}{ Education level } & None or primary school & $21 / 107$ & 19.4 & $12 / 114$ & 10.7 & $12 / 107$ & 11.4 & $7 / 114$ & 6.5 \\
\hline & Secondary school & $195 / 506$ & 38.5 & $138 / 578$ & 23.9 & $167 / 506$ & 33.0 & $88 / 578$ & 15.3 \\
\hline & High school & $352 / 790$ & 44.5 & $312 / 898$ & 34.8 & $295 / 790$ & 37.4 & $224 / 898$ & 25.0 \\
\hline \multirow{5}{*}{$\begin{array}{l}\text { Monthly income per } \\
\text { consumption unit }\end{array}$} & $\leq 1000 €$ & $152 / 283$ & 53.7 & $112 / 331$ & 33.9 & $123 / 283$ & 43.6 & $84 / 331$ & 25.4 \\
\hline & $1001-1500 €$ & $139 / 312$ & 44.7 & $118 / 351$ & 33.7 & $118 / 312$ & 37.8 & $89 / 351$ & 25.3 \\
\hline & $1501-2000 €$ & $112 / 259$ & 43.4 & $87 / 312$ & 27.8 & $96 / 259$ & 37.1 & $61 / 312$ & 19.6 \\
\hline & $2001-3000 €$ & $96 / 283$ & 34.0 & 109/339 & 32.1 & $80 / 283$ & 28.2 & $66 / 339$ & 19.4 \\
\hline & $>3000 €$ & $67 / 266$ & 25.3 & $37 / 256$ & 14.4 & $57 / 266$ & 21.3 & $20 / 256$ & 7.9 \\
\hline \multirow{4}{*}{$\begin{array}{l}\text { Religious affiliation and } \\
\text { practice }\end{array}$} & Regular religious practice & $69 / 211$ & 32.5 & $86 / 376$ & 22.9 & $47 / 211$ & 22.3 & $54 / 376$ & 14.4 \\
\hline & Irregular religious practice & $95 / 228$ & 41.4 & $86 / 318$ & 27.1 & $75 / 228$ & 33.0 & $65 / 318$ & 20.4 \\
\hline & Religious affiliation, no practice & $148 / 401$ & 36.8 & $128 / 425$ & 30.2 & $123 / 401$ & 30.6 & $80 / 425$ & 18.8 \\
\hline & Neither practice nor affiliation & $257 / 562$ & 45.6 & $163 / 471$ & 34.5 & $229 / 562$ & 40.7 & $121 / 471$ & 25.7 \\
\hline \multicolumn{10}{|l|}{$\begin{array}{l}\text { Variables related to sexual } \\
\text { biography }\end{array}$} \\
\hline \multirow[t]{4}{*}{ Couple status } & No relationship & 219/303 & 72.4 & $147 / 419$ & 35.1 & $214 / 303$ & 70.8 & $126 / 419$ & 30.0 \\
\hline & Love affair & $126 / 162$ & 77.5 & 119/186 & 63.8 & $115 / 162$ & 71.1 & 105/186 & 56.4 \\
\hline & Non-cohabiting couple & $57 / 88$ & 65.5 & $36 / 77$ & 46.2 & $51 / 88$ & 58.3 & $30 / 77$ & 38.9 \\
\hline & Cohabiting couple & $165 / 851$ & 19.4 & $162 / 907$ & 17.8 & $94 / 851$ & 11.0 & $59 / 907$ & 6.5 \\
\hline \multirow{3}{*}{$\begin{array}{l}\text { Lifetime number of couple } \\
\text { relationships }\end{array}$} & Never any couple relationship & $259 / 323$ & 80.3 & $151 / 258$ & 58.3 & $248 / 323$ & 76.8 & $133 / 258$ & 51.6 \\
\hline & One couple relationship & $150 / 670$ & 22.3 & $168 / 920$ & 18.3 & $95 / 670$ & 14.2 & $98 / 920$ & 10.6 \\
\hline & $\geq 2$ couple relationships & $159 / 410$ & 38.7 & $144 / 411$ & 35.0 & $131 / 410$ & 32.0 & $89 / 411$ & 21.7 \\
\hline \multirow{4}{*}{$\begin{array}{l}\text { Number of sexual partners } \\
\text { during the last } 5 \text { years }\end{array}$} & Never had sex & $2 / 17$ & 12.1 & $5 / 57$ & 8.6 & $0 / 17$ & 0.0 & $0 / 57$ & 0.0 \\
\hline & None & $34 / 114$ & 29.8 & $40 / 244$ & 16.5 & $31 / 114$ & 26.7 & $27 / 244$ & 10.9 \\
\hline & One & $154 / 796$ & 19.4 & $212 / 1004$ & 21.1 & $86 / 796$ & 10.9 & $111 / 1004$ & 11.0 \\
\hline & Two or more & $377 / 476$ & 79.2 & $206 / 284$ & 72.3 & $357 / 476$ & 75.1 & $182 / 284$ & 64.2 \\
\hline \multirow{2}{*}{$\begin{array}{l}\text { Self-perceived sexual } \\
\text { orientation }\end{array}$} & Heterosexual & $522 / 1336$ & 39.1 & $454 / 1545$ & 29.4 & $436 / 1336$ & 32.6 & $313 / 1545$ & 20.3 \\
\hline & Bisexual & $8 / 13$ & 62.9 & $6 / 11$ & 51.9 & $8 / 13$ & 62.9 & $6 / 11$ & 51.9 \\
\hline
\end{tabular}


Table 1 Prevalence of the intention of protection against HIV and the adoption of a condom-based approach for protection against HIV by subgroups. Paris metropolitan area, France, 2010 (Continued)

\begin{tabular}{|c|c|c|c|c|c|c|c|c|c|}
\hline & Homosexual & $35 / 40$ & 87.4 & $2 / 8$ & 24.5 & $28 / 40$ & 70.7 & $0 / 8$ & 0.0 \\
\hline & Not answered & $2 / 14$ & 15.5 & $2 / 26$ & 5.8 & $2 / 14$ & 14.8 & $1 / 26$ & 4.7 \\
\hline \multicolumn{10}{|c|}{ Variables related to communication about sexuality and perceptions of HIV } \\
\hline \multirow[t]{6}{*}{ Intimate social network } & 0 person & $124 / 557$ & 22.3 & $77 / 546$ & 14.1 & $94 / 557$ & 16.9 & $31 / 546$ & 5.7 \\
\hline & $1-2$ person(s) & $179 / 375$ & 47.7 & $167 / 525$ & 31.8 & $140 / 375$ & 37.4 & $127 / 525$ & 24.2 \\
\hline & 3-5 persons & $131 / 237$ & 55.3 & $143 / 288$ & 49.6 & $121 / 237$ & 50.9 & $110 / 288$ & 38.2 \\
\hline & 6-25 persons & $60 / 73$ & 81.8 & $23 / 64$ & 36.2 & $57 / 73$ & 77.6 & $17 / 64$ & 25.9 \\
\hline & Anyone & $40 / 66$ & 60.4 & $24 / 59$ & 40.1 & $35 / 66$ & 53.4 & $14 / 59$ & 24.3 \\
\hline & Not answered & $33 / 94$ & 35.3 & $29 / 107$ & 27.5 & $27 / 94$ & 28.5 & $21 / 107$ & 19.6 \\
\hline \multirow{2}{*}{$\begin{array}{l}\text { Perceived susceptibility to } \\
\text { HIV infection }\end{array}$} & No & $350 / 1054$ & 33.2 & $263 / 1221$ & 21.6 & $290 / 1054$ & 27.6 & $171 / 1221$ & 14.0 \\
\hline & Yes & $217 / 349$ & 62.3 & $200 / 369$ & 54.2 & $184 / 349$ & 52.7 & $149 / 369$ & 40.4 \\
\hline \multirow[t]{2}{*}{ Stigma toward HIV+ people } & No & $269 / 520$ & 51.7 & $243 / 641$ & 37.8 & $233 / 520$ & 44.7 & $180 / 641$ & 28.1 \\
\hline & Yes & 298/883 & 33.8 & $220 / 948$ & 23.3 & $242 / 883$ & 27.4 & $140 / 948$ & 14.8 \\
\hline \multicolumn{10}{|c|}{ Variables related to HIV prevention behaviours } \\
\hline \multirow[t]{3}{*}{ Use of condoms } & Never/unknown & $18 / 248$ & 7.4 & $45 / 524$ & 8.6 & $0 / 248$ & 0.1 & $4 / 524$ & 0.7 \\
\hline & $>5$ years ago & $81 / 502$ & 16.1 & $100 / 520$ & 19.2 & $45 / 502$ & 8.9 & $42 / 520$ & 8.1 \\
\hline & $\leq 5$ years ago & $468 / 654$ & 71.7 & $318 / 545$ & 58.3 & $429 / 654$ & 65.7 & $274 / 545$ & 50.3 \\
\hline \multirow[t]{2}{*}{ Voluntary HIV testing } & No & $322 / 943$ & 34.1 & 193/1123 & 17.2 & 255/943 & 27.1 & $127 / 1123$ & 11.3 \\
\hline & Yes & $246 / 460$ & 53.5 & $270 / 467$ & 57.8 & $219 / 460$ & 47.7 & $193 / 467$ & 41.3 \\
\hline
\end{tabular}

more likely to do so than women (36.8 vs. $27.3 \%$, $p<0.001)$. Only 4 in 3006 interviewees $(0.1 \%)$ didn't answer that question.

Being a foreigner from Europe or other parts of the world except Africa was associated with a less frequent intention of protection in men $(\mathrm{aOR}=0.49$ and $\mathrm{aOR}=$ 0.38 respectively, Table 2, men, model 1) and women $(\mathrm{aOR}=0.28$ and $\mathrm{aOR}=0.15$ respectively, Table 2 , women, model 1), but these differences were no longer significant in the fully adjusted models (Table 2, models 2). Conversely, we observed that, in men, the addition of socioeconomic variables revealed strong and significant associations between being French of SSA origin or being foreigner from SSA and the intention of protection, that persisted in the full model, with aOR increasing up to 2.66 (95\% CI $=[1.34-5.31])$ and 3.43 (95 \% CI = [1.66-7.13]), respectively (Table 2 , men, model 2 ). We observed a similar result in women, but for SSA foreigners only with aOR increasing up to 2.94 (95 \% CI $=[1.65-5.23]$, Table 2, women, model 2).

French women of Maghrebi origin and foreign Maghrebi women were both less likely to declare an intention of protection against HIV than the majority population when adjusting for age $(\mathrm{aOR}=0.35$ and $\mathrm{aOR}=0.22$ respectively, Table 2, women, model 1) but these two associations were no longer statistically significant when adjusting for the others variables (Table 2, women, model
2). No significant differences were ever observed for the corresponding male populations.

In univariate analysis, people who practice a religion were less likely to declare protecting themselves from HIV (OR of regular practice for both sex $=0.68,95 \%$ $\mathrm{CI}=[0.56-0.84])$ but this association disappeared when variables related to the sexual biographies were added to the model (Table 2, models 2).

Interestingly, the association between voluntary HIV testing and the intention of protection differed notably between genders in multivariate analysis. In women, testing was associated with more intention of protection $(\mathrm{aOR}=$ 1.90, $95 \% \mathrm{CI}=[1.44-2.52]$, Table 2, women, model 2) while men having been voluntarily tested for HIV expressed less intention to protect themselves $(\mathrm{aOR}=$ 0.58, $95 \% \mathrm{CI}=[0.40-0.83]$, Table 2, men, model 2). Additionally, the strength of the associations between condom use or intimate social network and the intention of protection were more important in men than in women.

\section{Adoption of a condom-based approach for protection against HIV}

Among the 2994 HIV negative people who had answered the question about the intention of protection, $680(22.7 \%)$ declared using condoms steadily to protect themselves from HIV (Table 1), more often men 
Table 2 Factors associated with the intention of protection against HIV according to the sex, Paris metropolitan area, France, 2010

\begin{tabular}{|c|c|c|c|c|c|c|c|c|c|}
\hline \multirow{3}{*}{ Variable } & \multirow{3}{*}{ Category } & \multicolumn{4}{|l|}{ Men } & \multicolumn{4}{|c|}{ Women } \\
\hline & & \multicolumn{2}{|c|}{ Model $1^{a}$} & \multicolumn{2}{|c|}{ Model $2^{b}$} & \multicolumn{2}{|c|}{ Model $1^{\mathrm{a}}$} & \multicolumn{2}{|c|}{ Model $2^{b}$} \\
\hline & & $\mathrm{aOR}$ & $95 \% \mathrm{Cl}$ & $\mathrm{aOR}$ & $95 \% \mathrm{Cl}$ & $\mathrm{aOR}$ & $95 \% \mathrm{Cl}$ & $\mathrm{aOR}$ & $95 \% \mathrm{Cl}$ \\
\hline \multirow[t]{9}{*}{ Migration origin } & French with Fr. parents (ref.) & 1 & - & 1 & - & 1 & - & 1 & - \\
\hline & French of Sub-Saharan origin & 1.39 & {$[0.80,2.41]$} & 2.66 & {$[1.34,5.31]^{* *}$} & 0.94 & {$[0.56,1.56]$} & 1.62 & {$[0.84,3.10]$} \\
\hline & Sub-Saharan foreigner & 1.48 & {$[0.71,3.08]$} & 3.43 & {$[1.66,7.13]^{* * *}$} & 1.50 & {$[0.93,2.43]$} & 2.94 & {$[1.65,5.23]^{* * *}$} \\
\hline & French of Maghrebi origin & 0.63 & {$[0.34,1.17]$} & 1.58 & {$[0.79,3.16]$} & 0.35 & {$[0.22,0.54]^{* * *}$} & 0.77 & {$[0.42,1.39]$} \\
\hline & Maghrebi foreigner & 0.81 & {$[0.45,1.45]$} & 1.49 & {$[0.54,4.10]$} & 0.22 & {$[0.10,0.52]^{* * *}$} & 0.57 & {$[0.23,1.43]$} \\
\hline & French of European origin & 0.89 & {$[0.44,1.81]$} & 1.08 & {$[0.51,2.25]$} & 0.69 & {$[0.43,1.10]$} & 0.97 & {$[0.55,1.69]$} \\
\hline & European foreigner & 0.49 & {$[0.26,0.93]^{*}$} & 1.77 & {$[0.86,3.66]$} & 0.58 & {$[0.34,1.00]^{*}$} & 1.02 & {$[0.52,2.03]$} \\
\hline & French of other origin & 0.87 & {$[0.53,1.42]$} & 1.88 & {$[0.83,4.24]$} & 0.80 & {$[0.53,1.22]$} & 1.37 & {$[0.83,2.25]$} \\
\hline & Other foreigner & 0.38 & {$[0.17,0.86]^{*}$} & 0.99 & {$[0.25,3.95]$} & 0.15 & {$[0.06,0.41]^{* * *}$} & 0.46 & {$[0.16,1.28]$} \\
\hline \multirow{4}{*}{$\begin{array}{l}\text { Religious affiliation } \\
\text { and practice }\end{array}$} & Regular religious practice & & & 1.04 & {$[0.56,1.95]$} & & & 1.02 & {$[0.66,1.57]$} \\
\hline & Irregular religious practice & & & 1.15 & {$[0.73,1.82]$} & & & 0.90 & {$[0.64,1.28]$} \\
\hline & Relig. affiliation, no practice & & & 0.98 & {$[0.65,1.50]$} & & & 1.00 & {$[0.70,1.42]$} \\
\hline & Neither practice nor affil (ref) & & & 1 & - & & & 1 & - \\
\hline \multirow[t]{4}{*}{ Couple status } & No relationship & & & 3.06 & {$[1.84,5.08]^{* * *}$} & & & 3.30 & {$[2.17,5.01]^{* * *}$} \\
\hline & Love affair & & & 2.04 & {$[1.15,3.59]^{*}$} & & & 3.00 & {$[1.95,4.63]^{* * *}$} \\
\hline & Non-cohabiting couple & & & 3.65 & {$[1.73,7.69]^{* * *}$} & & & 1.49 & {$[0.79,2.83]$} \\
\hline & Cohabiting couple (ref.) & & & 1 & - & & & 1 & - \\
\hline \multirow{3}{*}{$\begin{array}{l}\text { Lifetime number of } \\
\text { couple relationships }\end{array}$} & Never any couple relationship & & & 2.44 & {$[1.39,4.29]^{* *}$} & & & 2.63 & {$[1.70,4.09]^{* * *}$} \\
\hline & One couple relationship (ref.) & & & 1 & - & & & 1 & - \\
\hline & $\geq 2$ couple relationships & & & 1.08 & {$[0.76,1.54]$} & & & 1.44 & {$[1.03,2.03]^{*}$} \\
\hline \multirow{4}{*}{$\begin{array}{l}\text { Number of sexual } \\
\text { partners during } \\
\text { the last } 5 \text { years }\end{array}$} & Never had sex & & & 0.40 & {$[0.03,5.59]$} & & & 0.11 & {$[0.03,0.45]^{* *}$} \\
\hline & None & & & 1.47 & {$[0.72,3.00]$} & & & 0.85 & {$[0.53,1.35]$} \\
\hline & One & & & 1 & - & & & 1 & - \\
\hline & Two or more & & & 1.95 & {$[1.26,3.03]^{* *}$} & & & 2.05 & {$[1.44,2.92]^{* * *}$} \\
\hline \multirow{4}{*}{$\begin{array}{l}\text { Self-perceived sexual } \\
\text { orientation }\end{array}$} & Heterosexual (ref.) & & & 1 & - & & & 1 & - \\
\hline & Bisexual & & & 0.49 & {$[0.19,1.24]$} & & & 0.54 & {$[0.28,1.06]$} \\
\hline & Homosexual & & & 2.28 & {$[0.48,10.8]$} & & & 0.37 & {$[0.07,1.91]$} \\
\hline & Not answered & & & 0.22 & {$[0.07,0.71]^{*}$} & & & 0.69 & {$[0.25,1.86]$} \\
\hline \multirow[t]{6}{*}{ Intimate social network } & 0 person (ref.) & & & 1 & - & & & 1 & - \\
\hline & 1-2 person(s) & & & 1.54 & {$[1.02,2.34]^{*}$} & & & 0.90 & {$[0.67,1.20]$} \\
\hline & $3-5$ persons & & & 1.94 & {$[1.21,3.11]^{* *}$} & & & 1.53 & {$[1.05,2.22]^{*}$} \\
\hline & 6-25 persons & & & 5.15 & {$[2.34,11.3]^{* * *}$} & & & 0.91 & {$[0.48,1.71]$} \\
\hline & Anyone & & & 2.95 & {$[1.32,6.58]^{* *}$} & & & 2.09 & {$[0.85,5.15]$} \\
\hline & Not answered & & & 1.31 & {$[0.64,2.68]$} & & & 0.99 & {$[0.59,1.68]$} \\
\hline \multirow{2}{*}{$\begin{array}{l}\text { Perceived susceptibility } \\
\text { to HIV infection }\end{array}$} & No (ref.) & & & 1 & - & & & 1 & - \\
\hline & Yes & & & 1.98 & {$[1.18,3.33]^{* *}$} & & & 1.55 & {$[1.07,2.22]^{*}$} \\
\hline \multirow{2}{*}{$\begin{array}{l}\text { Stigma toward HIV+ } \\
\text { people }\end{array}$} & No (ref.) & & & 1 & - & & & 1 & - \\
\hline & A bit - a lot & & & 0.63 & {$[0.44,0.91]^{*}$} & & & 0.70 & {$[0.55,0.89]^{* *}$} \\
\hline \multirow[t]{2}{*}{ Use of condoms } & Never/unknown (ref.) & & & 1 & - & & & 1 & - \\
\hline & $>5$ years ago & & & 2.86 & {$[1.62,5.05]^{* * *}$} & & & 1.84 & {$[1.17,2.90]^{* *}$} \\
\hline
\end{tabular}


Table 2 Factors associated with the intention of protection against HIV according to the sex, Paris metropolitan area, France, 2010 (Continued)

\begin{tabular}{|c|c|c|c|c|c|}
\hline & $\leq 5$ years ago & 17.8 & {$[8.83,35.9]^{* * *}$} & 4.34 & {$[2.73,6.91]^{* * *}$} \\
\hline \multirow[t]{2}{*}{ Voluntary HIV testing } & No (ref.) & 1 & - & 1 & - \\
\hline & Yes & 0.58 & {$[0.40,0.83]^{* *}$} & 1.90 & {$[1.44,2.52]^{* * *}$} \\
\hline
\end{tabular}

(29.5\%) than women $(18.3 \%, p<0.001)$. Most of those who chose condoms as a means of protection had expressed an intention of protection (26/688, $96.2 \%)$ but, inversely, $29.3 \%(275 / 937)$ of people who intended to protect themselves from HIV selected other means than condom use.

In men, being French of Maghrebi origin or foreign from out of Africa and Europe was associated with less recourse to a condom-based approach of protection when adjusted for age $(\mathrm{aOR}=0.39$ and $\mathrm{aOR}=0.25$ respectively, Table 3, men, model 1 ). These associations disappeared when we adjusted for variables regarding sexual biographies (Table 3, men, model 2). No significant association with a SSA origin or nationality was observed.

In women, in the age-adjusted models (Table 3, women, model 1), being French of Maghrebi origin and being a Maghrebi foreigner were both associated with a lower probability of declaring a condom-based approach for protection: $\mathrm{aOR}=0.28,95 \% \mathrm{CI}=[0.14-0.53]$ and $\mathrm{aOR}=0.31,95 \% \mathrm{CI}=[0.14-0.69]$, respectively. It was also the case for foreign women from Europe or other parts of the world except for Africa $(\mathrm{aOR}=0.47$ and $\mathrm{aOR}=0.32$ respectively) but, as in men, these differences were no longer significant in the fully adjusted models (Table 3, women, model 2).

Globally, adjustment variables were associated with the recourse to a condom-based approach of protection in the same way than they were with the intention of protection, except that we did not observe here any significant association with the perceived susceptibility to HIV infection, nor with voluntary HIV testing, among men.

\section{Discussion}

Our results show that people's HIV prevention attitudes differ according to their country of origin. First, models adjusting only for demographic features show that Maghrebi women and French women of Maghrebi origin try less to protect themselves from HIV, and rely less on condom for protection against HIV than French women with French parents. This result suggests that Maghrebi women are insufficiently reached by prevention campaigns. It may be because they have a limited access to the messages, e.g. because they are not an actual target group in a context where most new HIV infections in foreign women are diagnosed in women originating from Sub-Saharan Africa. It may also be due to messages being insufficiently tailored for these persons, that are then not clearly understood or don't translate into a change in attitude or behaviour, e.g. because of the women's status in some of the Maghrebi immigrant subgroups that hinders communication about sexuality or because they distance themselves from risk groups. As noted already 10 years ago in France [27], Maghrebi population -originally from countries where the HIV epidemic is concentrated in high-risk groups [37] - still miss prevention interventions as compared to the rest of the general population in France. This may result from a low level of acculturation in France, which is a barrier to health-related communication and specifically prevention messages [38] and deserve the reinforcement of specific, targeted, information and prevention programs. These associations between Maghrebi citizenship or origin and a reduced intention for protection against HIV disappear when variables reflecting the women's socioeconomic situation and sexual biography are included into the models: it seems that these characteristics outweigh migration origin and/or - at least partially - explain the origin-related differences previously observed.

A very similar situation was observed among the few foreign women from non-African and non-European countries included in our sample. The small number of these women (a heterogeneous population structured in small communities in the Parisian population) and, also, the fact that our sample missed the neighbourhoods inhabited by Asian communities (although numerous in the Parisian area) make this result difficult to interpret and infer.

The second foremost result is that, in fully adjusted models, foreigners from SSA and French men of SSA origin declare more often that they do something to protect themselves from HIV than the French with French parents. This can be due to recent prevention campaigns specifically addressed to SSA populations in France (and/or those in their countries of origin) $[13,15]$ since perceiving oneself as being part of a risk group influence prevention behaviours $[21,22]$. It can also be related to certain forms of positive discrimination in individual HIV prevention promotion performed by health 
Table 3 Factors associated with the adoption of a condom-based approach for protection against HIV according to sex, Paris metropolitan area, France, 2010

\begin{tabular}{|c|c|c|c|c|c|c|c|c|c|}
\hline \multirow{3}{*}{ Variable } & \multirow{3}{*}{ Category } & \multicolumn{4}{|l|}{ Men } & \multicolumn{4}{|c|}{ Women } \\
\hline & & \multicolumn{2}{|c|}{ Model $1^{a}$} & \multicolumn{2}{|c|}{ Model $2^{b}$} & \multicolumn{2}{|c|}{ Model $1^{a}$} & \multicolumn{2}{|c|}{ Model $2^{b}$} \\
\hline & & $\mathrm{aOR}$ & $95 \% \mathrm{Cl}$ & $\mathrm{aOR}$ & $95 \% \mathrm{Cl}$ & $\mathrm{aOR}$ & $95 \% \mathrm{Cl}$ & $\mathrm{aOR}$ & $95 \% \mathrm{Cl}$ \\
\hline \multirow[t]{9}{*}{ Migration origin } & French with Fr. parents (ref.) & 1 & - & 1 & - & 1 & - & 1 & - \\
\hline & French of Sub-Saharan origin & 0.65 & {$[0.28,1.51]$} & 0.79 & {$[0.22,2.81]$} & 0.75 & {$[0.41,1.38]$} & 1.62 & {$[0.68,3.88]$} \\
\hline & Sub-Saharan foreigner & 0.59 & {$[0.23,1.55]$} & 1.38 & {$[0.38,4.93]$} & 0.64 & {$[0.35,1.16]$} & 0.93 & {$[0.40,2.18]$} \\
\hline & French of Maghrebi origin & 0.39 & {$[0.19,0.79]^{* *}$} & 0.75 & {$[0.34,1.67]$} & 0.28 & {$[0.14,0.53]^{* * *}$} & 0.65 & {$[0.30,1.42]$} \\
\hline & Maghrebi foreigner & 0.58 & {$[0.32,1.02]$} & 1.15 & {$[0.45,2.95]$} & 0.31 & {$[0.14,0.69]^{* *}$} & 0.81 & {$[0.23,2.87]$} \\
\hline & French of European origin & 1.21 & {$[0.56,2.62]$} & 2.00 & {$[0.84,4.75]$} & 0.64 & {$[0.36,1.13]$} & 1.13 & {$[0.52,2.46]$} \\
\hline & European foreigner & 0.48 & {$[0.23,1.01]$} & 1.73 & {$[0.58,5.12]$} & 0.47 & {$[0.24,0.92]^{*}$} & 0.93 & {$[0.31,2.82]$} \\
\hline & French of other origin & 0.58 & {$[0.32,1.07]$} & 0.89 & {$[0.35,2.26]$} & 0.73 & {$[0.42,1.27]$} & 1.28 & {$[0.62,2.63]$} \\
\hline & Other foreigner & 0.25 & {$[0.10,0.61]^{* *}$} & 0.68 & {$[0.16,2.91]$} & 0.32 & {$[0.13,0.82]^{*}$} & 2.34 & {$[0.78,7.00]$} \\
\hline \multirow{4}{*}{$\begin{array}{l}\text { Religious affiliation } \\
\text { and practice }\end{array}$} & Regular religious practice & & & 0.93 & {$[0.51,1.71]$} & & & 0.67 & {$[0.38,1.18]$} \\
\hline & Irregular religious practice & & & 1.33 & {$[0.81,2.17]$} & & & 0.86 & {$[0.51,1.44]$} \\
\hline & Relig. affiliation, no practice & & & 0.89 & {$[0.52,1.54]$} & & & 0.57 & {$[0.40,0.82]^{* *}$} \\
\hline & Neither practice nor affil (ref) & & & 1 & - & & & 1 & - \\
\hline \multirow[t]{4}{*}{ Couple status } & No relationship & & & 8.18 & {$[4.46,15.0]^{* * *}$} & & & 14.5 & {$[8.35,25.2]^{* * *}$} \\
\hline & Love affair & & & 4.01 & {$[1.91,8.45]^{* * *}$} & & & 9.94 & {$[5.57,17.7]^{* * *}$} \\
\hline & Non-cohabiting couple & & & 5.80 & {$[2.43,13.8]^{* * *}$} & & & 4.32 & {$[1.96,9.51]^{* * *}$} \\
\hline & Cohabiting couple (ref.) & & & 1 & - & & & 1 & - \\
\hline \multirow{3}{*}{$\begin{array}{l}\text { Lifetime number of } \\
\text { couple relationships }\end{array}$} & Never any couple relationship & & & 2.24 & {$[1.16,4.35]^{*}$} & & & 1.97 & {$[1.31,2.96]^{* *}$} \\
\hline & One couple relationship (ref.) & & & 1 & - & & & 1 & - \\
\hline & $\geq 2$ couple relationships & & & 1.14 & {$[0.68,1.90]$} & & & 1.47 & {$[1.01,2.13]^{*}$} \\
\hline \multirow{4}{*}{$\begin{array}{l}\text { Number of sexual } \\
\text { partners during the } \\
\text { last } 5 \text { years }\end{array}$} & Never had sex & & & n. e. & & & & n.e. & \\
\hline & No partner since 5 years & & & 0.81 & {$[0.37,1.76]$} & & & 0.64 & {$[0.36,1.13]$} \\
\hline & One partner last 5 years (ref.) & & & 1 & - & & & 1 & - \\
\hline & $\geq 2$ partners last 5 years & & & 6.48 & {$[4.17,10.1]^{* * *}$} & & & 4.37 & {$[3.04,6.28]^{* * *}$} \\
\hline \multirow{4}{*}{$\begin{array}{l}\text { Self-perceived sexual } \\
\text { orientation }\end{array}$} & Heterosexual (ref.) & & & 1 & - & & & 1 & - \\
\hline & Bisexual & & & 0.64 & {$[0.18,2.26]$} & & & 0.88 & {$[0.40,1.95]$} \\
\hline & Homosexual & & & 0.94 & {$[0.29,3.08]$} & & & n.e. & \\
\hline & Not answered & & & 0.16 & {$[0.04,0.59]^{* *}$} & & & 1.15 & {$[0.42,3.16]$} \\
\hline \multirow[t]{6}{*}{ Intimate social network } & 0 person (ref.) & & & 1 & - & & & 1 & - \\
\hline & 1-2 person(s) & & & 1.37 & {$[0.83,2.28]$} & & & 1.37 & {$[0.83,2.27]$} \\
\hline & $3-5$ persons & & & 2.60 & {$[1.25,5.39]^{*}$} & & & 2.39 & {$[1.42,4.00]^{* * *}$} \\
\hline & 6-25 persons & & & 3.85 & {$[1.46,10.1]^{* *}$} & & & 1.78 & {$[0.80,3.98]$} \\
\hline & Anyone & & & 1.40 & {$[0.66,2.97]$} & & & 1.67 & {$[0.75,3.73]$} \\
\hline & Not answered & & & 0.90 & {$[0.36,2.28]$} & & & 1.48 & {$[0.68,3.20]$} \\
\hline \multirow{2}{*}{$\begin{array}{l}\text { Perceived susceptibility } \\
\text { to HIV infection }\end{array}$} & No (ref.) & & & 1 & - & & & 1 & - \\
\hline & Yes & & & 1.42 & {$[0.86,2.34]$} & & & 1.46 & {$[1.03,2.05]^{*}$} \\
\hline \multirow{2}{*}{$\begin{array}{l}\text { Stigma toward HIV+ } \\
\text { people }\end{array}$} & No (ref.) & & & 1 & - & & & 1 & - \\
\hline & A bit - a lot & & & 0.63 & {$[0.42,0.95]^{*}$} & & & 0.51 & {$[0.36,0.72]^{* * *}$} \\
\hline
\end{tabular}


Table 3 Factors associated with the adoption of a condom-based approach for protection against HIV according to sex, Paris metropolitan area, France, 2010 (Continued)

\begin{tabular}{lllll}
\hline Voluntary HIV testing & Never/unknown (ref.) & 1 & - & - \\
& $>5$ years ago & 1.10 & {$[0.72,1.70]$} & 1 \\
& & & {$[1.11,2.51]^{*}$} \\
\hline
\end{tabular}

ne not estimated (no individual in the category has adopted a condom-based approach for protection against HIV)

${ }^{*} p<0.05$; ${ }^{* *} p<0.01$; ${ }^{* * *} p<0.001$

${ }^{a}$ : model adjusted on age

${ }^{b}$ : model adjusted on age, level of education and monthly household income per consumption unit

care workers, similarly to what has been previously described for HIV testing [22, 26]. Alternatively it may be related to a social desirability bias that is known to be more frequent in cultures based on oral tradition such as those in SSA [39]. It is worth noting that this more frequent declaration of protection did not translate into a more frequent recourse to the use of condoms as a means of protection in any of these 3 subgroups. If, generally speaking, most of the individual characteristics associated with the intention to protect oneself against HIV were also associated with a condom-based approach, this was less true for the origin from SSA, possibly because people in this category opt more for other means such as HIV testing, as described in the Paris area [40]. Concerning women, it has been shown that they are used to resort to other means of protection than condoms only [34]. These various results confirm the need for a culture-specific approach of HIV prevention and in the interpretation of results of HIV related surveys [11, 22, 24, 26, 40].

Globally, our results show that French people with foreign origin are in an intermediate situation between French people with French parents and foreigners from the same continent. This likely reflects the acculturation process [4] and, more precisely, the maintenance - or the re-appropriation - of cultural norms inherited from the culture of origin. Given the definition of the different categories of French people with foreign origins used here, we cannot go further in this cultural interpretation and distinguish between the situation of people born French in France to foreign parents and those of the immigrants who acquired the French citizenship (each representing about half of the above mentioned categories). Conversely, even if limited in their interpretation, our results are the first quantitative results ever published in France in a representative, population-based sample about the situation of the French people of foreign origin in the field of HIV prevention (and of HIV epidemiology in general). We think that replicating such approaches in other European countries would be very useful at the time when European public health institutions, such as the European Centre for Disease Prevention and Control, emphasize that prevention efforts should not only aim at the general population but also at groups such as immigrants or minorities [41, 42]. Their results may usefully precede and/or trigger the development of HIV prevention interventions targeted to immigrants at a larger scale or with more significant resources.

Looking at covariables, some results deserve being discussed as they confirm or infirm previous findings. We found that people with a religious affiliation were less likely to intend for protecting themselves against HIV, similarly to what has been observed previously in France $[40,43]$ but this influence was not observable when we adjusted for variables related to sexual biography. This suggests that the impact of religion on means of prevention may be limited to (or mediated by) an influence on the sexual biography even if further analysis would be necessary to explore this pathway. Conversely, our results clearly show that affective and marital status has a major impact on the individual's definition of protection means for oneself and plea, with others, for a systematic adjustment for the couple status to study these HIV prevention strategies, which was not done in the above mentioned study [43]. We also observed that individuals stigmatizing HIV+ people were less likely to intend to protect themselves against HIV and to opt for condombased approaches for protection. This result was expected since self-protective attitudes and stigma share common determinants such as perceived severity of AIDS and perceived self-susceptibility to HIV infection [44]. Nevertheless the fact that the stigma and the perceived susceptibility to HIV infection are both independent factors in our models and a restricted mediation analysis (not presented) showing that only $12.3 \%$ of the effect of stigma on the intention of protection is mediated by the perceived susceptibility, suggest that stigma and perceived susceptibility are related with intended prevention behaviours mostly through unconnected pathways, unlike suggested elsewhere [45]. The importance of other determinants, such as stereotypes, has not been evaluated in our study and it is difficult to do so in a quantitative study like this one. Obviously, qualitative studies make invaluable contributions to a deep and comprehensive knowledge of the various determinants of these attitudes and behaviours [11].

This study presents some limitations. Our sample is restricted to Paris metropolitan area and our results 
cannot be extrapolated to the whole France. However since this region bears the most important part of the HIV burden in mainland France, our conclusions are pertinent to help in the management of the epidemic in France but also in foreign settings with similar features [46]. The attrition rate between 2005 and 2010 might have influenced the representativeness of the sample, but has few chance to affect the relationships between variables in a sample of this size [47] and we must remind here that the non-reinterviewed people have been replaced by people selected by the same random procedure (with a quite good response rate for a populationbased survey). Also the sampling design didn't allow for reaching very vulnerable populations such as homeless people, among which immigrants are overrepresented [48] and we interviewed only people speaking French when non-French speaking people accounted for $6.8 \%$ of individuals contacted for the study. If those people could have been included and interviewed in SIRS, the difference between French people and foreigners might have been accentuated (by construction, our sample does not allow us either to test the influence of speaking French or not). The questionnaire of the SIRS surveys didn't allow for the investigation of ways of transmission of HIV other than sexual and was also limited by the measurement of multipartnership over the last 5 years only, while it is usually calculated on the sexual activity of the previous year $[19,49]$. Also, several factors known to impact the strategies of protection against STDs were not measured, such as the social context in which a sexual relationship takes place [50]. Moreover, since our questions investigated attitudes and declared behaviours but not practices (e.g. the utilization of condoms during last sexual intercourses or according to different kinds of sexual partners), we are unable to evaluate the gap between intention and effective practices [19].

\section{Conclusion}

According to their migration origin, some foreigners and French people of foreign origin show attitudes and behaviours profiles regarding HIV protection that are different from those of the majority of the population in France. These differences argue for the importance of a culture-specific approach of HIV prevention. Even if some attention is currently paid for immigrants from SSA, our results draw attention to other fractions of the population that could still escape from prevention messages in France, especially people with Maghrebi origins. In this way, our results plea for further investigations (and a public health monitoring) of their situations in France.

\section{Additional file}

Additional file 1: Complete intermediate multivariate models.

Competing interests

The authors declare that they have no competing interests.

\section{Authors' contributions}

TK: has made substantial contributions to conception and design, acquisition of data, analysis and interpretation of data and has been involved in drafting the manuscript and revising it critically for important intellectual content. AL: has made substantial contributions to conception and design, or acquisition of data, analysis and interpretation of data and has been involved in drafting the manuscript and revising it critically for important intellectual content. VM: has been involved in drafting the manuscript and revising it critically for important intellectual content. DC: has been involved in drafting the manuscript and revising it critically for important intellectual content. PC: has made substantial contributions to conception and design, or acquisition of data, analysis and interpretation of data and have been involved in drafting the manuscript and revising it critically for important intellectual content. All authors have given final approval of the version to be published.

\section{Acknowledgements}

The 2010 data collection of the SIRS cohort study was supported by the Institute for Public Health Research (IRESP) and has also benefited from the support of the Mutualité Française. This study was part of a specific research project supported by the French National Agency for Research on AIDS (ANRS) and Sidaction. Paul Duke and Nas Sabrie deserve special thanks for their proofreading.

Received: 28 October 2013 Accepted: 10 July 2015

Published online: 20 August 2015

\section{References}

1. Jusot $F$, Silva J, Dourgnon $P$, Sermet $C$. Inégalités de santé liées à l'immigration en France: Effet des conditions de vie ou sélection à la migration ? Rev Econ. 2009;60:385-412.

2. Lassetter $\mathrm{JH}$, Callister LC. The impact of migration on the health of voluntary migrants in western societies. J Transcult Nurs. 2009;20:93-104.

3. Bhopal RS. Ethnicity, Race, and Health in Multicultural Societies: Foundations for Better Epidemiology, Public Health and Health Care. New York: Oxford University Press; 2007. p. 150-81.

4. Meston CM, Ahrold T. Ethnic, gender, and acculturation influences on sexual behaviors. Arch Sex Behav. 2010;39:179-89.

5. Yu J. Young people of Chinese origin in western countries: a systematic review of their sexual attitudes and behaviour. Heal Soc Care Community. 2010;18:117-28.

6. Weiss UK, Tillman KH. Risky sexual behaviors among Hispanic young adults in South Florida: nativity, age at immigration and gender differences. Perspect Sex Reprod Heal. 2009;41:202-9.

7. Noiriel G, Borne D. Population, Immigration et Identité Nationale En France, XIXe-XXe Siècle. Paris: Hachette; 1992. p. 43-83.

8. Régnard C. La population étrangère résidant en France. Infos Migr 2009:1-4

9. European Centre for Disease Prevention and Control: Migrant Health. Epidemiology of HIV and AIDS in Migrant Communities and Ethnic Minorities in EU / EEA Countries. Stockholm: European Centre for Disease Prevention and Control: Migrant Health; 2010.

10. Lelkes O, Zólyomi E. Poverty and Social Exclusion of Migrants in the European Union. In: Policy Briefs. 2011. p. 1-17.

11. McMahon T, Ward PR. HIV among immigrants living in high-income countries: a realist review of evidence to guide targeted approaches to behavioural HIV prevention. Syst Rev. 2012;1:56.

12. Cazein F, Le Strat $Y$, Pillonel J, Lot F, Bousquet V, Pinget $R$, et al. Dépistage du VIH et découvertes de séropositivité, France, 2003-2010. Bull Epidemiol Hebd. 2011:43-44:446-54.

13. Ministère de la Santé et des Sports. Plan National de Lutte Contre Le VIH/ SIDA et Les IST 2010-2014. Paris: Ministère de la Santé et des Sports; 2010. p. 19-33. 
14. Direction Générale de la Santé. Programme National de Lutte Contre Le VIH-Sida En Direction Des Étrangers/migrants Vivant En France 2004-2006. Paris: Direction Générale de la Santé; 2004. p. 1-64.

15. INPES - Sida. [http://www.inpes.sante.fr/10000/themes/sida/index.asp]

16. Pauti MD, Simonnot N, Estecahandy P. [Development of actions for the prevention of HIV, hepatitis and sexually transmitted infections among immigrants consulting in the doctors of the world "Missions France"]. Médecine Mal Infect. 2009;39:191-5.

17. Lert F, Pialoux G. Rapport Mission RDRs - Prévention et Réduction Des Risques Dans Les Groupes À Haut Risque vis-à-vis Du VIH et Des IST. 2010. p. 187-91.

18. Bajos N. Social factors and the process of risk construction in HIV sexual transmission. AIDS Care. 1997;9:227-37.

19. Bajos N, Ducot B, Spencer B, Spira A. Sexual risk-taking, socio-sexual biographies and sexual interaction: Elements of the French national survey on sexual behaviour. Soc Sci Med. 1997:44:25-40.

20. Dean HD, Fenton KA. Addressing social determinants of health in the prevention and control of HIV/AIDS, viral hepatitis, sexually transmitted infections, and tuberculosis. Public Health Rep. 2010;125 Suppl 4:1-5.

21. Pourette D. Migratory Paths, Experiences of HIV/AIDS, and Sexuality: African Women Living withHIV/AIDS in France. Fem Econ. 2008;14:149-81.

22. Marsicano E, Lydié N, Bajos N. "Migrants from over there" or "racial minority here"? Sexual networks and prevention practices among sub-Saharan African migrants in France. Cult Health Sex. 2013;15:819-35.

23. Cherabi K, Fanget D. Le VIH/sida En Milieu Migrant Arabo-Musulman En France. Paris; 1997

24. Beltzer N, Fénies K, Halfen S, Lert F, Levu S, Lydié N. Les Populations Africaines d'lle-de-France Face Au VIH/sida - Connaissances, Attitudes, Croyances et Comportements. Paris: INPES; 2007.

25. Bozon M, Doré V, Fassin D, Grémy I, Halfen S, Solier N, et al. Sida, Immigration et Inégalités: Nouvelles Réalités, Nouveaux Enjeux. Paris: Agence Nationale de Recherches sur le Sida; 2002.

26. Lapostolle A, Massari V, Chauvin P. Time since the last HIV test and migration origin in the Paris metropolitan area, France. AIDS Care. 2011;23:1117-27.

27. Messiah A, Rey D, Obadia Y, Rotily M, Moatti JP. HIV testing, knowledge, attitudes, beliefs, and practices among minorities: pregnant women of North-African origin in southeastern France. J Natl Med Assoc. 1998;90:87-92.

28. Chauvin P, Parizot I. Les Inégalités Sociales et Territoriales de Santé Dans L'agglomération Parisienne. Une Analyse de La Cohorte Sirs (2005). Saint-Denis La Plaine: Délégation interministérielle à la ville; 2009. p. 1-105.

29. Vallée J, Cadot E, Grillo F, Parizot I, Chauvin P. The combined effects of activity space and neighbourhood of residence on participation in preventive health-care activities: The case of cervical screening in the Paris metropolitan area (France). Health Place. 2010;16:838-52.

30. Roustit C, Campoy E, Renahy E, King G, Parizot I, Chauvin P. Family social environment in childhood and self-rated health in young adulthood. BMC Public Health. 2011;11:949.

31. Vallée J, Cadot E, Roustit C, Parizot I, Chauvin P. The role of daily mobility in mental health inequalities: the interactive influence of activity space and neighbourhood of residence on depression. Soc Sci Med. 2011;73:1133-44.

32. Grillo F, Vallée J, Chauvin P. Inequalities in cervical cancer screening for women with or without a regular consulting in primary care for gynaecological health in Paris, France. Prev Med. 2012;54:259-65.

33. European Centre for Disease Prevention and Control. Improving HIV Data Comparability in Migrant Populations and Ethnic Minorities in EU/EEA/ EFTA Countries. Stockholm: European Centre for Disease Prevention and Control; 2011

34. Beltzer N, Lagarde M, Wu-Zhou X, Vongmany N, Grémy I. Les Connaissances, Attitudes, Croyances et Comportements Face Au Sida En Île-de-France - Evolutions 1992, 1994, 1998, 2001, 2004. Paris; 2005

35. Beltzer N, Fénies K, Halfen S, Lert F, Le Vu S, Lydié N. Les Populations Africaines d'lle-de-France Face Au VIH/sida - Connaissances, Attitudes, Croyances et Comportements. Paris; 2007

36. Bajos N, Bozon M. Enquête Sur La Sexualité En France - Pratiques, Genre et Santé. Paris: Editions La Découverte; 2008.

37. Abu-raddad LJ, Hilmi N, Mumtaz G, Benkirane M, Ayodeji F, Riedner G. Epidemiology of HIV infection in the Middle East and North Africa. AIDS. 2010:24 Supl 2:S5-23.
38. Kinsler JJ, Lee SJ, Sayles JN, Newman PA, Diamant A, Cunningham W. The impact of acculturation on utilization of HIV prevention services and access to care among an at-risk Hispanic population. J Heal Care Poor Underserved. 2009:20:996-1011.

39. Michalon C. Histoire de Différences, Différence D’histoires. Sepia: Saint-Maur; 2002.

40. Massari V, Lapostolle A, Cadot E, Parizot I, Dray-Spira R, Chauvin P. Gender, socio-economic status, migration origin and neighbourhood of residence are barriers to HIV testing in the Paris metropolitan area. AIDS Care. 2011;23:1609-18

41. European Centre for Disease Prevention and Control. A Comprehensive Approach to HIV/STI Prevention in the Context of Sexual Health in the EU/ EEA. Stockholm: European Centre for Disease Prevention and Control; 2013.

42. European Centre for Disease Prevention and Control. Assessing the Burden of Key Infectious Diseases Affecting Migrant Populations in the EU/EEA. Stockholm: European Centre for Disease Prevention and Control; 2014.

43. Lagarde E, Enel C, Seck K, Gueye-Ndiaye A, Piau J-P, Pison G, et al. Religion and protective behaviours towards AIDS in rural Senegal. AIDS. 2000:14:2027-33.

44. Allard R. Beliefs about AIDS as determinants of preventive practices and of support for coercive measures. Am J Public Heal. 1989;79:448-52.

45. Riley GA, Baah-Odoom D. Do stigma, blame and stereotyping contribute to unsafe sexual behaviour? A test of claims about the spread of HIV/AIDS arising from social representation theory and the AIDS risk reduction model. Soc Sci Med. 2010;71:600-7.

46. Halfen S, Embersin-Kyprianou C, Grémy I. Suivi de l'infection à VIH/sida en lle-de-France. In: Bull Santé l'ORS Ile-de-France. 2008. p. 1-8.

47. Nemes S, Jonasson JM, Genell A, Steineck G. Bias in odds ratios by logistic regression modelling and sample size. BMC Med Res Methodol. 2009;9:56.

48. Laporte A, Chauvin P. La Santé Mentale et Les Addictions Chez Les Personnes sans Logement Personnel d'lle-de-France. Samenta. Paris: Observatoire du samu social et Inserm; 2010. p. 226.

49. Mercer $\mathrm{CH}$, Copas AJ, Sonnenberg P, Johnson AM, McManus S, Erens B, et al. Who has sex with whom? Characteristics of heterosexual partnerships reported in a national probability survey and implications for STI risk. Int J Epidemiol. 2009;38:206-14

50. Bajos N, Marquet J. Research on HIV sexual risk: Social relations-based approach in a cross-cultural perspective. Soc Sci Med. 2000;50:1533-46.

\section{Submit your next manuscript to BioMed Central and take full advantage of:}

- Convenient online submission

- Thorough peer review

- No space constraints or color figure charges

- Immediate publication on acceptance

- Inclusion in PubMed, CAS, Scopus and Google Scholar

- Research which is freely available for redistribution

Submit your manuscript at www.biomedcentral.com/submit
C Biomed Central 